\title{
Categorization of Data Mining Systems and Data Secrecy Towards Wireless Sensor Networks
}

\section{Shoban Babu Sriramoju ${ }^{1}$}

${ }^{1}$ Project Manager, Kenexcel Software Pvt. Ltd., India, ${ }^{1}$ Research Scholar, Kalinga University, India

\begin{abstract}
The reliable database administration systems have actually been very vital assets for monitoring of a large corpus of data as well as particularly for reliable as well as efficient access of particular information from a huge collection whenever required. The expansion of data source monitoring systems has actually also contributed to recent huge gathering of all kind of info. This paper deals with the classification of data mining systems and also issues available in Data Mining.
\end{abstract}

Keywords : Data Mining, Classification, Association Rule, WSN

\section{INTRODUCTION}

We are in an age commonly described as the information age. In this details age, due to the fact that our company believe that info causes power and also success, as well as thanks to innovative modern technologies such as computer systems, satellites, etc., we have actually been collecting significant quantities of information. At first, with the advent of computer systems and implies for mass electronic storage space, we started gathering as well as keeping all kind of data, trusting the power of computer systems to help sort with this amalgam of details. Unfortunately, these large collections of data saved on diverse frameworks really swiftly came to be frustrating. This first turmoil has caused the creation of structured databases as well as database administration systems (DBMS). Today, we have much more info than we can deal with: from organisation deals and also scientific data, to satellite pictures, message reports as well as army intelligence. Information retrieval is just inadequate anymore for decision-making. Faced with massive collections of data, we have currently produced brand-new demands to aid us make better managerial options. These demands are automated summarization of data, removal of the "significance" of details stored, and the exploration of patterns in raw data.

\section{What kind of information are we collecting?}

We have actually been accumulating a myriad of data, from easy mathematical dimensions and also message records, to more intricate details such as spatial data, multimedia channels, and also hypertext files. Here is a non-exclusive list of a range of information accumulated in electronic form in data sources as well as in level documents.

- Organisation deals: Every deal in the business sector is (typically) "memorized" for perpetuity. Such transactions are generally time related and can be inter-business offers such as acquisitions, exchanges, financial, supply, and so on, or intra-business operations such as administration of in-house wares and possessions. Large chain store, for instance, thanks to the prevalent use upc code, store millions of transactions day-to-day standing for frequently 
terabytes of data. Storage space is not the significant issue, as the cost of hard disks is continuously going down, but the reliable use of the data in a practical timespan for competitive decision- making is absolutely the most vital problem to address for businesses that have a hard time to make it through in a highly affordable world.

- Scientific data: Whether in a Swiss nuclear accelerator lab counting particles, in the Canadian woodland researching readings from a grizzly bear radio collar, on a South Pole iceberg gathering data concerning nautical task, or in an American college examining human psychology, our culture is collecting gigantic quantities of clinical data that need to be analyzed. However, we can capture as well as save more brand-new data faster than we can examine the old data already gathered.

- Medical and personal data: From government demographics to personnel and also consumer files, huge collections of details are continually gathered concerning people and also teams. Governments, companies as well as organizations such as health centers, are stockpiling really vital quantities of individual data to help them handle human resources, better comprehend a market, or merely help clients. Regardless of the personal privacy concerns this sort of data often exposes, this information is gathered, utilized and even shared. When associated with other data this info can clarify customer behaviour and the like.

- Security video and photos: With the amazing collapse of camera costs, camera are coming to be common. Video tapes from monitoring video cameras are generally reused and also thus the web content is shed. However, there is a tendency today to store the tapes and even digitize them for future use and evaluation.
- Satellite noticing: There is a many variety of satellites around the globe: some are geo-stationary over an area, and some are orbiting around the Planet, however all are sending out a continuous stream of data to the surface. NASA, which regulates a lot of satellites, gets more data every second than what all NASA researchers and also designers can cope with. Several satellite images and also data are revealed as soon as they are received in the hopes that researchers can analyze them.

- Games: Our culture is collecting an incredible quantity of data as well as statistics regarding video games, players and professional athletes. From hockey scores, basketball passes and car-racing lapses, to swimming times, boxer's presses and chess positions, all the data are saved. Commentators and journalists are using this information for coverage, yet trainers and athletes would want to manipulate this data to improve performance as well as better understand opponents.

- Digital media: The expansion of affordable scanners, desktop computer camera as well as digital electronic cameras is just one of the root causes of the surge in digital media repositories. On top of that, lots of radio stations, tv networks as well as film studios are digitizing their audio and video clip collections to improve the monitoring of their multimedia assets. Organizations such as the NHL and also the NBA have already begun transforming their huge game collection into digital types.

- CAD and also Software design data: There are a multitude of Computer Assisted Style (CAD) systems for designers to make structures or designers to conceive system parts or circuits. These systems are creating a significant amount of data. Additionally, software program engineering is a source of 
considerable similar data with code, function libraries, items, etc., which need powerful devices for management as well as maintenance.

- Virtual Worlds: There are numerous applications using three-dimensional virtual rooms. These spaces and also the items they contain are defined with unique languages such as VRML. Preferably, these digital spaces are explained as though they can share objects and areas. There is an impressive quantity of virtual reality object and also space repositories available. Administration of these repositories in addition to content-based search as well as access from these repositories are still research study problems, while the dimension of the collections remains to expand.

- Text reports as well as memos (e-mail messages): Most of the communications within and also in between companies or research organizations and even personal people, are based on reports and memoranda in textual kinds often traded by e-mail. These messages are consistently kept in electronic kind for future usage and also reference creating awesome digital libraries.

- The Internet repositories: Since the beginning of the Web in 1993, records of all kind of formats, web content and description have actually been accumulated and also inter-connected with links making it the largest repository of data ever built. Regardless of its dynamic as well as disorganized nature, its heterogeneous particular, as well as its really typically redundancy as well as variance, the Net is the most important data collection consistently utilized for reference due to the broad selection of subjects covered as well as the limitless payments of sources and also publishers. Many believe that the Web will end up being the compilation of human knowledge.

\section{Data Mining and Understanding Exploration?}

With the substantial quantity of data kept in documents, databases, and also other databases, it is significantly vital, otherwise necessary, to develop effective means for evaluation as well as perhaps analysis of such data and also for the removal of intriguing understanding that can aid in decisionmaking.

Data Mining, additionally widely called Knowledge Discovery in Databases (KDD), describes the nontrivial removal of implied, formerly unidentified as well as potentially beneficial details from data in data sources. While data mining and also understanding discovery in databases (or KDD) are frequently treated as basic synonyms, data mining is really part of the expertise discovery process The complying with figure (Figure 1) reveals data mining as an action in an iterative understanding exploration procedure.

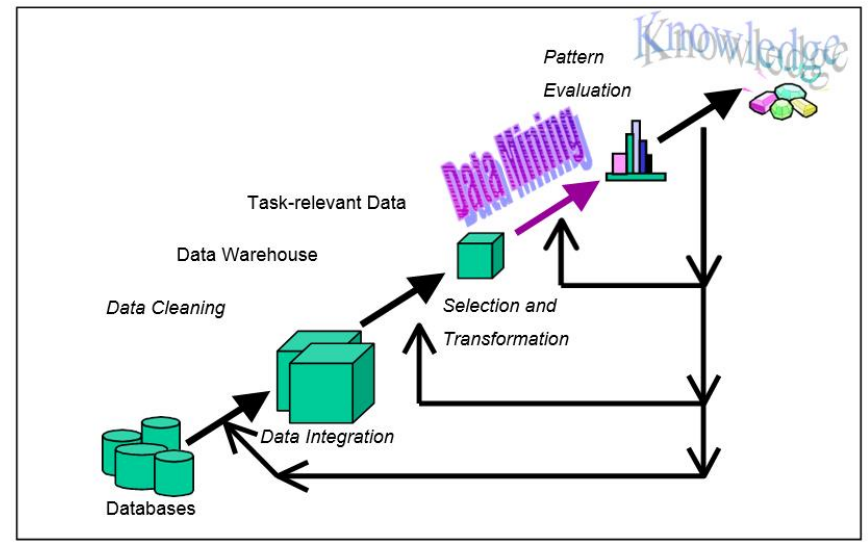

Figure 1: Data Mining is the core of Knowledge Discovery process

The Knowledge Discovery in Databases procedure consists of a couple of actions leading from raw data collections to some form of brand-new expertise. The iterative process contains the following steps: 
- Data cleaning: likewise known as data cleaning, it is a stage in which sound data and also unimportant data are eliminated from the collection.

- Data assimilation: at this stage, multiple data sources, often heterogeneous, might be incorporated in an usual resource.

- Data choice: at this action, the data pertinent to the evaluation is decided on as well as obtained from the data collection.

- Data improvement: likewise known as data loan consolidation, it is a phase in which the picked data is changed into types proper for the mining procedure.

- Data mining: it is the vital step in which smart techniques are related to extract patterns possibly beneficial.

- Pattern evaluation: in this action, purely fascinating patterns representing expertise are recognized based upon given actions.

- Understanding depiction: is the last phase in which the uncovered knowledge is visually stood for to the individual. This essential step makes use of visualization strategies to aid individuals understand and interpret the data mining results.

It prevails to combine a few of these steps together. As an example, data cleaning as well as data assimilation can be carried out together as a preprocessing phase to generate a data stockroom. Data choice as well as data transformation can also be integrated where the consolidation of the data is the outcome of the choice, or, when it comes to the instance of data stockrooms, the option is done on changed data.

The KDD is an iterative procedure. When the found knowledge exists to the individual, the assessment procedures can be improved, the mining can be more refined, new data can be picked or more transformed, or new data sources can be integrated, to get different, better results.

Data mining derives its name from the resemblances in between searching for useful information in a big data source and mining rocks for a vein of important ore. Both suggest either looking through a large quantity of material or ingeniously probing the product to precisely pinpoint where the worths reside. It is, nevertheless, a misnomer, since mining for gold in rocks is normally called "gold mining" as well as not "rock mining", hence by analogy, data mining need to have been called "expertise mining" instead. However, data mining came to be the approved customary term, and also extremely swiftly a fad that also eclipsed even more basic terms such as understanding exploration in data sources (KDD) that describe a more total procedure. Various other similar terms describing data mining are: data dredging, understanding removal and also pattern exploration.

The figure shows summarized leasings organized by film categories, after that a cross table of summed up rentals by film classifications as well as time (in quarters). The data dice gives the summed up rentals along three dimensions: category, time, and also city. A dice includes cells that keep worths of some aggregate measures (in this case rental counts), and unique cells that store summations along dimensions. Each dimension of the data dice includes a power structure of values for one characteristic.

\section{Is all that is discovered interesting and useful?}

Data mining permits the discovery of understanding possibly helpful and also unknown. Whether the knowledge discovered is new, helpful or interesting, is very subjective as well as relies on the application as well as the individual. It is specific that data 
mining can create, or discover, a huge number of patterns or guidelines. In some cases the variety of regulations can reach the millions. One can also think of a meta-mining phase to extract the large data mining results. To reduce the variety of patterns or policies discovered that have a high possibility to be non-interesting, one needs to put a dimension on the patterns. Nonetheless, this increases the trouble of completeness. The customer would certainly intend to uncover all rules or patterns, but only those that are interesting. The dimension of how intriguing a discovery is, commonly called interestingness, can be based on quantifiable objective elements such as validity of the patterns when tested on new data with some level of certainty, or on some subjective representations such as understandability of the patterns, novelty of the patterns, or effectiveness.

Discovered patterns can additionally be found fascinating if they verify or validate a hypothesis looked for to be verified or suddenly contradict a typical idea. This brings the problem of explaining what interests discover, such as meta-rule directed exploration that defines forms of guidelines prior to the exploration procedure, and also interestingness refinement languages that interactively query the outcomes for interesting take after the exploration phase. Typically, dimensions for interestingness are based on thresholds established by the customer. These limits specify the completeness of the patterns uncovered.

Identifying and also determining the interestingness of patterns and rules discovered, or to be uncovered, is essential for the evaluation of the mined expertise and the KDD procedure all at once. While some concrete dimensions exist, evaluating the interestingness of found expertise is still an essential research problem.

\section{How do we categorize data mining systems?}

There are lots of data mining systems readily available or being created. Some are specialized systems devoted to a given data resource or are constrained to minimal data mining performances, various other are much more versatile and thorough. Data mining systems can be categorized according to various criteria among other category are the following:

- Category according to the sort of data resource mined: this category categorizes data mining systems according to the kind of data dealt with such as spatial data, multimedia data, time-series data, message data, Net, etc.

- Classification according to the data design made use of: this classification classifies data mining systems based on the data design involved such as relational data source, object-oriented data source, data storage facility, transactional, etc.

- Category according to the king of knowledge discovered: this category classifies data mining systems based on the kind of knowledge uncovered or data mining capabilities, such as characterization, discrimination, organization, classification, clustering, etc. Some systems often tend to be thorough systems using several data mining capabilities together.

- Classification according to mining techniques used: Data mining systems utilize and also provide various techniques. This category categorizes data mining systems according to the data analysis method made use of such as machine learning, semantic networks, hereditary algorithms, stats, visualization, databaseoriented or data warehouse-oriented, etc. The category can additionally consider the level of customer interaction associated with the data mining procedure such as query-driven systems, interactive exploratory systems, or autonomous systems. 


\section{What are the issues in Data Mining?}

Data mining formulas symbolize methods that have often existed for years, yet have just recently been used as reputable and also scalable tools that time and also again outperform older classic analytical approaches. While data mining is still in its infancy, it is ending up being a pattern and common. Before data mining develops into a traditional, fully grown and also relied on self-control, many still pending concerns need to be addressed. A few of these issues are resolved listed below. Note that these concerns are not special and are not gotten at all.

Security and also social concerns: Safety is a crucial issue with any data collection that is shared and/or is planned to be utilized for calculated decision-making. In addition, when data is gathered for customer profiling, user behaviour understanding, associating personal data with other details, and so on, large quantities of sensitive and also personal info regarding people or companies is gathered as well as kept. This comes to be questionable provided the personal nature of some of this data and the prospective prohibited access to the information. In addition, data mining can reveal brand-new implied understanding regarding people or teams that could be against personal privacy policies, specifically if there is possible circulation of discovered info. Another concern that occurs from this issue is the proper use data mining. Because of the worth of data, data sources of all sorts of content are routinely sold, as well as due to the competitive advantage that can be attained from implied expertise found, some important info could be held back, while various other details could be extensively distributed as well as used without control.

User interface issues: The expertise discovered by data mining tools works as long as it is fascinating, and most of all understandable by the customer. Excellent data visualization eases the analysis of data mining results, in addition to aids users better comprehend their requirements. Many data exploratory analysis jobs are substantially facilitated by the capability to see data in a proper aesthetic discussion. There are numerous visualization ideas as well as proposals for reliable data graphical presentation. However, there is still much study to complete in order to acquire good visualization tools for large datasets that could be made use of to display and control mined knowledge. The major problems related to interface and also visualization are "screen real-estate", details rendering, as well as communication. Interactivity with the data as well as data mining results is vital given that it offers methods for the user to focus and also refine the mining jobs, as well as to visualize the discovered expertise from various angles and at various conceptual levels.

Mining methodology problems: These problems pertain to the data mining comes close to applied and their restrictions. Topics such as adaptability of the mining methods, the variety of data readily available, the dimensionality of the domain name, the broad evaluation demands (when known), the analysis of the expertise discovered, the exploitation of history knowledge as well as metadata, the control as well as handling of noise in data, and so on are all examples that can determine mining approach choices. As an example, it is commonly desirable to have different

data mining techniques offered because various approaches might perform in different ways relying on the data available. Furthermore, various approaches may match and solve customer's demands in different ways. 
Most algorithms presume the data to be noise-free. This is obviously a solid presumption. Many datasets include exceptions, void or incomplete details, etc., which may make complex, otherwise unknown, the evaluation process as well as in a lot of cases endanger the accuracy of the results. Consequently, data preprocessing (data cleaning and makeover) becomes crucial. It is typically seen as wasted time, however data cleaning, as time- consuming and also irritating maybe, is among one of the most essential stages in the understanding exploration procedure. Data mining techniques ought to be able to deal with noise in data or insufficient info.

More than the dimension of data, the dimension of the search room is even more definitive for data mining methods. The size of the search space is usually depending upon the variety of measurements in the domain space. The search room normally expands exponentially when the variety of dimensions rises. This is known as the curse of dimensionality. This "curse" influences so terribly the performance of some data mining approaches that it is becoming one of one of the most immediate issues to fix.

Efficiency problems: Many expert system and analytical approaches exist for data analysis and also analysis. Nevertheless, these techniques were often not created for the very large data sets data mining is taking care of today. Terabyte dimensions are common. This raises the concerns of scalability and effectiveness of the data mining methods when processing substantially huge data. Formulas with rapid and also medium-order polynomial complexity can not be of useful use for data mining. Linear algorithms are normally the norm. In very same theme, tasting can be made use of for mining as opposed to the entire dataset. Nonetheless, issues such as completeness and option of samples might occur. Various other subjects in the problem of efficiency are incremental upgrading, and also parallel shows. There is no question that similarity can aid solve the dimension issue if the dataset can be partitioned as well as the outcomes can be merged later on. Incremental updating is necessary for combining results from parallel mining, or updating data mining results when new data appears without having to re-analyze the complete dataset.

Data resource concerns: There are many issues related to the data sources, some are sensible such as the variety of data types, while others are thoughtful like the data excess issue. We definitely have an excess of data since we already have much more data than we can handle and we are still accumulating data at an also higher rate. If the spread of data source management systems has actually assisted boost the event of information, the introduction of data mining is absolutely urging more data harvesting. The present method is to accumulate as much data as possible currently and refine it, or attempt to process it, later. The issue is whether we are gathering the ideal data at the suitable quantity, whether we understand what we want to perform with it, and also whether we compare what data is necessary as well as what data is trivial. Pertaining to the practical problems connected to data resources, there is the topic of heterogeneous databases as well as the concentrate on varied complex data types. We are storing different kinds of data in a selection of databases. It is challenging to anticipate a data mining system to efficiently as well as effectively achieve good mining results on all kinds of data and resources. Various type of data and also sources may require distinct formulas as well as approaches. Presently, there is a concentrate on relational databases as well as data storage facilities, however other approaches require to be originated for other details facility data kinds. A flexible data mining device, for all type of 
data, might not be sensible. Additionally, the proliferation of heterogeneous data sources, at architectural and semantic degrees, presents important obstacles not only to the database community however additionally to the data mining area

\section{DATA SECRECY AND AUTHENTICATION}

We are making use of RC5 as block cipher for file encryption paired with OCB, so that both file encryption as well as verification are attained in just one pass, thus conserving in processing time and energy at sensing unit node. Sensor node makes use of security to provide data secrecy, however in some cases there is really little difference in successive analyses of a sensor node.We have actually made use of a nonce as a counter, which is made use of in file encryption, thus making sure each time different cipher text is produced. We have utilized 4 level of file encryption to give adaptive safety. Degree of security will be provided by ISA.

Replay Security and Quality Inspect: Replay defense is provided by using a monotonically enhancing counter value at both ends or utilizing a time stamp in the message. We have actually supplied the counter value in a package header that is made use of to safeguard replay strikes. A monotonically boosting counter of 32 bits is made use of at the both ends. Yet just the last 8 little bits is sent out in the package for saving transmission and also reception power. The entire procedure is given as adheres to

- Presume that both the receiver and sender are having the exact same counter value of 32 little bits each.

- Operation of RC5-OCB is used making use of a counter value of 32bits as well as MAC is acquired.
- While sending out, full MAC (32 little bits) is sent but we send out just LSB 8 bits of counter value, hence conserving transmission of 24 bits.

- Receiver calculates an expected counter (Cs) number depending upon the last connection/ synchronization.

- After obtaining a packet the receiver will certainly concatenate Cs (0-23) as well as got counter worth (8bits). It will apply RC5-OCB operation to get MAC as well as cipher message as well as if the MAC is exact same, the package is accepted, otherwise it will certainly increment $\mathrm{Cs}$ and afterwards try determining MAC thinking some package loss.

There must be some bound over increment and inspect strategy given up step5, which can be done by establishing a threshold worth that depends upon the network packet loss price. Also it can be minimized by application of flower filter. [2] supplies a LOFT SPACE procedure that advises sending of only 3bits in packet. It will not suit for broadcast interaction, so we have actually taken a counter value of 8 bits to make our plan suitable for unicast along with broadcast communication. To make this method a lot more resilient to replay strikes, base station can broadcast the routine counter worth.

\section{VII.CONCLUSION}

Data mining automates the procedure of discovering predictive info in big data sources. Questions that traditionally needed extensive hands- on analysis can currently be responded to straight from the data-swiftly. A case in point of a predictive trouble is targeted advertising. Data mining uses data on past advertising mailings to identify the targets most likely to maximize roi in future mailings. This paper provided the categorization of data mining systems as 
well as likewise problems readily available in Data Mining

\section{REFERENCES}

[1]. R. Simon, Overseen Analysis When the Variety Of Prospect Attributes (p) Substantially Goes beyond The Number of Cases (n), SIGKDD Explorations Unique Concern on Microarray Data Mining, Dec 2003

[2]. E. R. Tufte, The Aesthetic Present of Measurable Details", second Edition, Graphics Press, 2001.

[3]. Quinlan, J. Ross, C4.5: Programs for Artificial Intelligence, Morgan Kaufmann, 1993.

[4]. Witten, Ian and also Eibe Frank, Data Mining, Practical Artificial Intelligence Tools as well as Methods with Java Implementations, Morgan Kaufmann, 1999.

[5]. Weng-Keen Wong, Andrew Moore, Gregory Cooper, Michael Wagner, Rule-based Abnormality Pattern Discovery for Detecting Condition Break Outs, Proc. of the 18th National Seminar on Artificial Intelligence, 2002.

[6]. Shoban Babu Sriramoju, Azmera Chandu Naik, N.Samba Siva Rao, "Predicting The Misusability Of Data From Malicious Insiders" in "International Journal of Computer Engineering and Applications" Vol V, Issue II,Febrauary 2014 ISSN : 2321-3469 ]

[7]. Ajay Babu Sriramoju, Dr. S. Shoban Babu, "Analysis on Image Compression Using BitPlane Separation Method" in "International Journal of Information Technology and Management", Vol VII, Issue X, November 2014 ISSN : 2249-4510 ]

[8]. Shoban Babu Sriramoju, "Mining Big Sources Using Efficient Data Mining Algorithms" in "International Journal of Innovative Research in Computer and Communication Engineering"
Vol 2, Issue 1,January 2014 ISSN(online) : 23209801, ISSN(print) : 2320-9798 ]

[9]. Ajay Babu Sriramoju, Dr. S. Shoban Babu, "Study of Multiplexing Space and Focal Surfaces and Automultiscopic Displays for Image Processing" in "International Journal of Information Technology and Management" Vol V, Issue I, August 2013 ISSN : 2249-4510 ]

[10]. Dr. Shoban Babu Sriramoju, "A Review on Processing Big Data" in "International Journal of Innovative Research in Computer and Communication Engineering" Vol-2, Issue-1, January 2014 ISSN(online) : 2320-9801, ISSN(print) : 2320-9798 ]

[11]. Shoban Babu Sriramoju, Dr. Atul Kumar, “An Analysis around the study of Distributed Data Mining Method in the Grid Environment : Technique, Algorithms and Services" in "Journal of Advances in Science and Technology" Vol-IV, Issue No-VII, November 2012 ISSN : 2230-9659 ]

[12]. Shoban Babu Sriramoju, Dr. Atul Kumar, "An Analysis on Effective, Precise and Privacy Preserving Data Mining Association Rules with Partitioning on Distributed Databases" in "International Journal of Information Technology and management" Vol-III, Issue-I, August 2012 ISSN : 2249-4510 ]

\section{Cite this article as :}

Shoban Babu Sriramoju, "Categorization of Data Mining Systems and Data Secrecy Towards Wireless Sensor Networks", International Journal of Scientific Research in Science and Technology (IJSRST), Online ISSN : 2395-602X, Print ISSN : 2395-6011, Volume 6 Issue 2, pp. 818-826, March-April 2019. Available at doi : https://doi.org/10.32628/IJSRST207258 Journal URL : http://ijsrst.com/IJSRST207258 